\title{
Assessment the quality of apricots in the process of drying with neural networks and support vector machines
}

\author{
Martin Dejanov ${ }^{1, *}$, Darinka Ilieva-Stefanova ${ }^{1}$, and Iva Chelik $^{1}$ \\ ${ }^{1}$ University of Ruse, Department of Automation and Mechatronics, 8 Studentska Str., 7017 Ruse, Bulgaria
}

\begin{abstract}
The paper presents an analysis of the assessment the quality of apricots during the drying process using two types of classifires: ANNs and SVMs. The quality of apricots is categorized in three classes according to the color and b-carotene content through the process of drying. The classification is made by using 'CIE Lab' color model and spectral characteristics in the VIS range. Neural networks are BPN and PNN, and classifiers are kernel and linear SVM. The spectral characteristics are pre-processed with SNV, MSC, First derivative and PCA. According to the results for color features, BPN and SVM with "rbf" kernel have the best performance while PNN has the worst performance. When using spectral characteristics the BPN network performs well: $\mathrm{e}_{\mathrm{avg}}=4.1 \%$ and $\mathrm{e}_{\max }=12.1 \%$ but the SVM linear $\left(\mathrm{e}_{\mathrm{avg}}=\right.$ $3.4 \%, \mathrm{e}_{\max }=5.3 \%$ ) and SVM with "rbf" kernel ( $\left.\mathrm{e}_{\mathrm{avg}}=2.4 \%, \mathrm{e}_{\max }=5.2 \%\right)$ classifiers have better results. As a conclusion, it could be said that classifiers using spectral features perform well with errors at about $2-5 \%$. Classification with color features is an alternative method, which is less complex, cheaper and with acceptable errors.
\end{abstract}

\section{INTRODUCTION}

It is well known that the quality is a critical issue facing the modern food industry because consumers always expect food products with excellent quality and reasonable price. In order to meet consumers' expectations to maintain food quality, the food manufacturers also require some suitable detection techniques and methods to ensure the quality of their products. However, the current traditional detection techniques and methods such as sensory evaluation or physical and chemical approaches are time-consuming, arduous and expensive. Therefore, rapid, nondestructive, and effective quality assessment techniques are needed. As an alternative to traditional methods for evaluation of food quality, noncontact optical methods based on analysis of color images, spectrophotometric and hyperspectral analysis are most perspective in the recent years. On the other hand, data mining is the process of automatic discovery of useful information in databases. Data mining techniques are useful for quality assessment tasks, particularly those that perform classification tasks [1-8]. These techniques use well-known methods of machine learning, pattern recognition, and statistics to find patterns that uncover hidden and useful information in data sets. Many successful applications used these techniques for food quality classification according to the characteristics of a product [9-12].

Some of the non-invasive techniques for predicting the food characteristics are done through computer vision and spectral analysis in a combination with artificial neural networks (ANN).
The application of thermal treatments in the food industry is one of the main stages to obtain a good quality product, whether applied in the pre-treatment or transformation stage. A feedback-type ANN was used to evaluate the concentration and temperature in a solution of fructooligosaccharides in parameters such as mass, moisture, volume, and solids in dehydrated yacon cubes [11]. In another study, a multilayer perceptron with a layer at the entrance with four neurons and an exit layer with two neurons was used to predict percentages of water loss and solids gain in the osmotic dehydration of aubergines [13]. Azadbakht et al.[14] found that for the determination of dehydration parameters of potato cubes, such as energy and exergy efficiency and the use of energy, the use of ANN provided better response approaches than the traditional mathematical models. On the other side, a study conducted by Khawas et al., [15] evaluated the influence of temperature, thickness and type of pretreatment, texture of the color and texture during vacuum dehydration of banana (MusaABB).

Amoriello et al. [16] study the quality of apricots. They demonstrated the possibility of sorting fresh apricots into uniform ripening classes using Vis and VisNIR spectrometers. Obtained results highlight how molecular spectroscopy coupled with multivariate analysis and statistical algorithms can identify and sort homogenous fruit batches with similar characteristics. Khojastehnazhand et al. [17] classified apricot fruits based on maturity levels and size by the use of image processing. The apricots were classified into three

\footnotetext{
* Corresponding author: mdejanov@uni-ruse.bg
} 
maturity stages (i.e. unripe, ripe, and overripe) and the volume was estimated. They said that the LDA and QDA could categorize the apricots with the accuracy of 0.904 and 0.923 , respectively based on color features. Results showed that the algorithm can properly classify the fruits using the image properties of apricots. Sani et al. [18] show the potential of using FT-NIR spectroscopy for the rapid and non-destructive measurement of the moisture, water activity, firmness and SO2 content in sulphureddried apricots. They demonstrate that the major quality parameters of sulphured-dried apricots can be measured in a short time by FT-NIR spectroscopy without any need for the sample preparation. Camps et al. [19] studied the ability of portable Near Infrared Spectroscopy to determine apricot fruit quality. Their calibration models allow determination of different apricot's quality features with variable precisions. They claim that NIRS technology could be applicable to apricot quality evaluation.

\section{Problem Formulation}

Apricots are cultivated world-wide mainly for their high-quality fruit, which is consumed fresh, processed by the food industry, or preserved by drying. Fruit quality is a combination of physical and chemical characteristics accompanied by sensory properties (appearance, texture, taste and aroma), nutritional values, chemical compounds, mechanical properties, and functional properties. The comprehensive term "quality" comprises of a number of parameters of the drying material, in a mid-state or after the completion of the drying process. These parameters are important for characterization and prediction of the quality of dried product. The quality related properties could be grouped into the following: structural (density, poriosity), optical (color, appearance), textural (compession test, stress relaxation), sensorial (aroma, taste), thermal (glassy, cristaline), nutritional (vitamins, proteins). The most promising methods are the optical methods. The phisicochemical changes that occur during the drying seem to affect the quality properties of the dehydrated product. The color of the material changes during dehydration not only due to evaportion of the surface water but also due to certain reactions, such as enzymatic browning, non-enzymatic browning and caramelization reactions. These reactions may be undesirable for many products as well as apricots. Thus, the examination of color and some internal content like b-carotene in the process of apricot drying is important.

\section{Problem Solution}

The aim of this research is to classify apricots in the process of drying in three different categories (classes) according to the color change and content of b-carotene. The quality of the apricots is classified in three main color categories - apricots with typical color, apricots with half-dried light-brown color and full-brown color apricots. The corresponding classes for $b$-carotene content are as follow: $210 \mathrm{mg} / \mathrm{kg}, 93.5 \mathrm{mg} / \mathrm{kg}$ and
$41.7 \mathrm{mg} / \mathrm{kg}$. To evaluate the quality of apricots in the process of drying, attributes such as color and spectal characteristics are used. The color and spectral characteristics are measured with Ocean Optics QE65000 spectrophotometer during the process of drying of the apricots. The results are processed using data mining techniques. The classification is done by using color features obtained from the 'CIE Lab' color model and spectral characteristics obtained in a VIS range (200-1000nm).

\subsection{Sample preparation and feature extraction}

The apricot samples were obtained from local markets in the city of Ruse (Bulgaria). The species of apricots is „New Jersey”. They were cut in two pieces and dried in the oven with a fixed temperature of $\mathrm{T}=65 \mathrm{o}$ and drying air speed of $\mathrm{v}=2 \mathrm{~m} / \mathrm{s}$. The drying process of apricots is shown on Fig.1.

Color and spectral features are obtained by Ocean optics - "QE65000" spectrophotometer with its ability to measure color. The features (color or spectrum) are measured in three specific points of a drying process: before the drying process begins, with 55\% moisture content and with $22 \%$ moisture content. These three batches of samples are used as groups (classes) in the process of drying. The following numbers of samples (points from the surface of apricots) are measured for the first, second and third class: 96, 61, and 128. The color and spectrum are measured form the apricot surface. In this study the three parameters: L, a, b, of 'CIE Lab' color model are used as an input parameters to the classifiers.

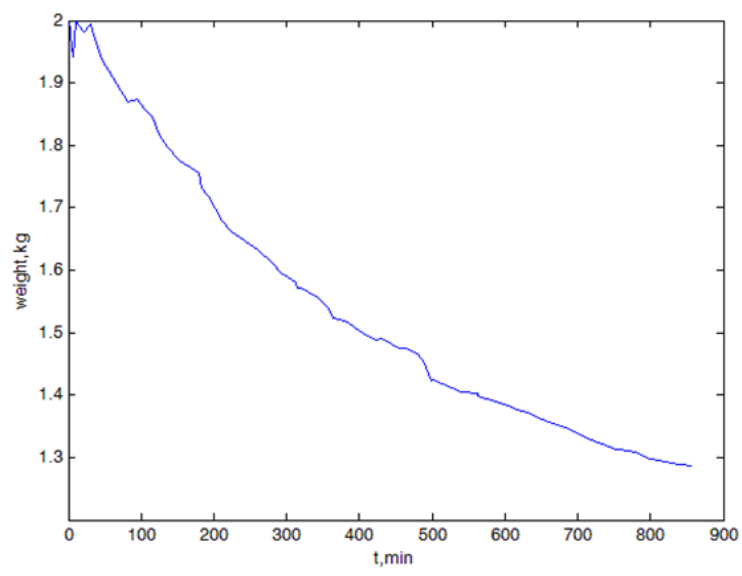

Fig. 1. The drying process of apricots

The spectral characteristics are obtained in the same way of the drying process as color features, and some pre-processing methods are applied to spectral data before it can be used for model preparation. These methods are standard normal variate (SNV), multiplicative scatter correction (MSC) and first derivative (Savitzky-Golay). After that, PCA is applied to the data. The Principal Component Analysis (PCA) is applied to the spectral characteristics in order to obtain an exploratory data analysis. PCA is a frequently 
employed unsupervised pattern recognition technique based on the linear combination of optimally-weighted observed variables (principal components) enabling data dimensionality reduction, while retaining maximum variability of the data. In this study, the first three principal components are used.

\subsection{Classification models}

In this study, two types of models are structured. The first model is based on neural networks: BPN and PNN, and the second - on the support vector machines - SVM (radial basis unction) and SVM (linear). Training and testing of neural networks and Support Vector Machine (SVM) classifiers is done in a "Matlab ${ }^{\text {TM" }}$ environment.

\subsubsection{Performance measures}

A classification task maps (in other words, classifies) a data item into one of several predefined classes based on data variables. To perform that, data mining uses machine learning to train algorithms, and uses the training data to build a classification model that is capable of predicting the class of unknown samples. One way to measure the performance of the classification model is by accuracy, which is the overall number of correct classifications, divided by the number of instances in the validation dataset. Some metrics are calculated based on the confusion matrix values. The matrix values are true positive (TP) for samples correctly classified as positive; true negative (TN) for samples correctly classified as negative; false negative (FN) for the positive samples that were classified as negative; and false positive (FP) for negative samples that are classified as positive. Using matrix values, it is possible to establish a performance measures like this bellow:

$$
e_{i}=\frac{F N_{i}}{\left(T P_{i}+F N_{i}\right)}
$$

$\mathrm{e}_{\mathrm{i}}$ gives the relative part of objects from some class $\mathrm{i}$, which are assigned incorrectly to other classes $k=1$..N, where $\mathrm{FNi}$ is the number of elements from the $\mathrm{i}$-th class classified incorrectly to other classes, TPi is the number of correctly classified elements from the i-th class.

$$
g_{i}=\frac{F P_{i}}{\left(T P_{i}+F P_{i}\right)}
$$

$\mathrm{g}_{\mathrm{i}}$ gives the relative part of objects from other classes, which are assigned to class $i$, where FPi is the number of elements from other classes, assigned to the i-th class.

$$
e_{o}=\frac{\sum_{i=1}^{N} F N_{i}}{\left(\sum_{i=1}^{N} T P_{i}+\sum_{i=1}^{N} F N_{i}\right)}
$$

$e_{o}$ (classification error rate) gives the relative part of all incorrectly classified objects where $\mathrm{N}$ is the number of classes.

\subsubsection{Neural Networks}

Multilayer Perceptron (MLP) is a classifier inspired by the way the human brain stores and processes knowledge. Neural networks can model real world complex relationships being able to predict unknown sample classes. Given a training set the neural network can learn the classification between the classes yi based on input data xi. The most commonly used neural network is a Multilayer Perceptron (MLP). The MLP can be used for linear and nonlinear modelling of inputoutput data. MLP trained with backpropagation algorithm is called BPN.

Other popular neural network is PNN. Probabilistic neural network (PNN) is closely related to Parzen window pdf estimator. A PNN consists of several subnetworks, each of which is a Parzen window pdf estimator for each of the classes. The input nodes are the set of measurements. The second layer consists of the Gaussian functions formed using the given set of data points as centres. The third layer performs an average operation of the outputs from the second layer for each class. The fourth layer performs a vote, selecting the largest value. The associated class label is then determined.

\subsubsection{Support Vector Machines}

Support Vector Machines (SVM) is a classifier which aims to find a hyperplane with maximum margin to separate the classes of data. A linear SVM is a classifier that searches the hyperplane with the widest margin. The goal is to maximize the margin of this hyperplane, maximizing the distance between the samples in its limit. In non-separable cases it is necessary to add slack variables and a kernel trick to separate data. The kernel trick is a commonly used technique to solve linearly inseparable problems. The kernel function performs a non-linear mapping into feature space. In this study, we used the Gaussian Radial Basis Function (RBF) kernel, defined as the following equation:

$$
k\left(x_{i}, x_{j}\right)=e^{-\left(\frac{x_{i}-x_{j}{ }^{2}}{2 \sigma^{2}}\right)}
$$

\subsection{Classification results}

In this study, cross-validation "k-fold" method will be used. The cross-validation gives the model an opportunity to test on multiple splits so we can get a better idea on how the model will perform on unseen data. In order to train and test our model using crossvalidation, we will use a cross-validation value of 5 .

\subsubsection{Quality assessment using ANN with color features}

The quality assessment of apricots in three classes by color features is based on two types of artificial neural networks: BPN and PNN.

BPN neural network designed for color feature classification is presented on Fig.2. Input layer has three 
inputs corresponding to each of the color model components (L, a, b) shown on Fig.3.

The output layer defines three classes (three quality groups), with the corresponding grouping being encoded through 0 - for not belonging to a class or 1- for belonging to a class. Because of that, the output layer has three neurons. Activation function is 'tangsig' and other parameters are as follows: epoches $=1000$ and $\mu=$ 0.001 .

The network training process includes a backpropagation algorithm. In the case of the BPN network, training is within 8 epochs. One of the best training, validation and testing is presented in Fig. 4. The average error over $\mathrm{k}$-folds is $15.1 \%$ and the confusion matrix of the biggest error is given in Table 1.

After that, input data is presented to train PNN neural network. It does not need validation but evaluates its perormance by the test data. The average error over kfolds is $54.5 \%$ and the confusion matrix of the biggest error is given in Table 2 .

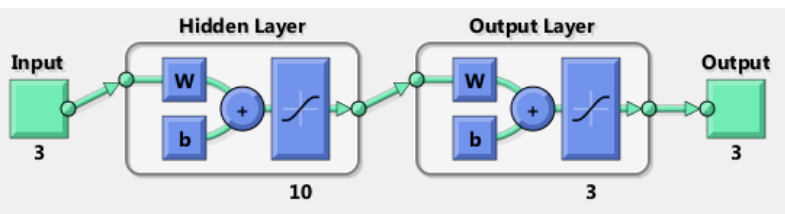

Fig. 2. BPN neural network for color feature classification

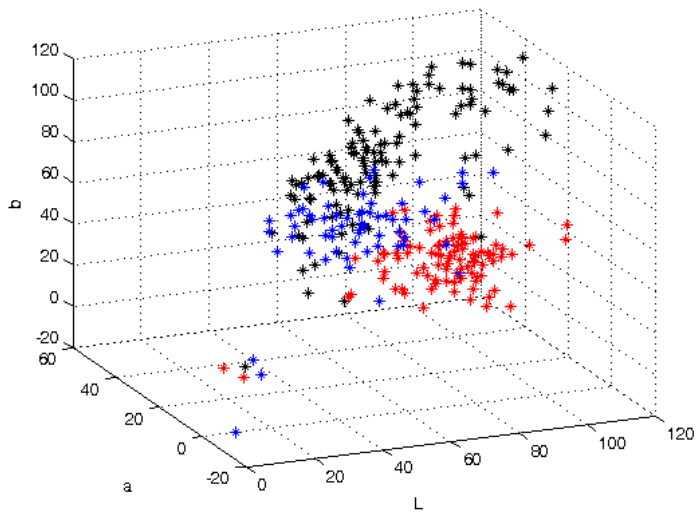

Fig. 3. Distribution of three classes in the 'Lab' color model

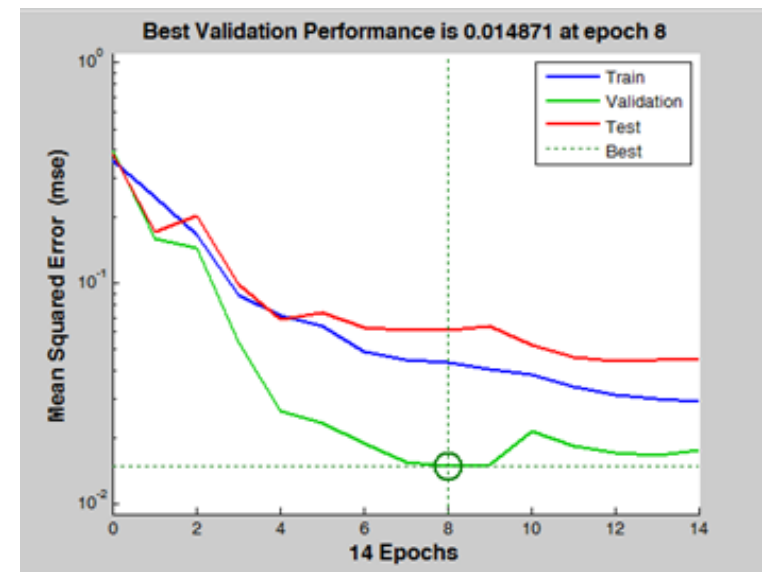

Fig. 4. Training, validation and testing of the best fold for BPN with color features
Table 1. The confusion matrix of the biggest error for BPN with color features.

\begin{tabular}{|c|c|c|c|c|c|c|}
\hline \multirow{2}{*}{\multicolumn{2}{|c|}{ BPN }} & \multicolumn{3}{|c|}{ True classes } & \multicolumn{2}{|c|}{ errors } \\
\hline & & 1 & 2 & 3 & $\mathbf{g}_{\mathbf{i}}$ & $\mathbf{e}_{\mathrm{i}}$ \\
\hline \multirow{3}{*}{ 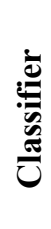 } & 1 & 1 & 2 & 1 & 18.1 & 6.9 \\
\hline & 2 & 0 & 0 & 0 & 20 & 36 \\
\hline & 3 & 19 & 2 & 23 & 8.3 & 8.3 \\
\hline \multicolumn{2}{|c|}{ All } & 20 & 12 & 24 & \multicolumn{2}{|c|}{$e_{0}=57.1 \%$} \\
\hline
\end{tabular}

Table 2. The confusion matrix of the biggest error for PNN with color features

\begin{tabular}{|c|c|c|c|c|c|c|}
\hline \multirow{2}{*}{\multicolumn{2}{|c|}{ PNN }} & \multicolumn{3}{|c|}{ True classes } & \multicolumn{2}{|c|}{ errors } \\
\hline & & 1 & 2 & 3 & $\mathbf{g}_{\mathbf{i}}$ & $\mathbf{e}_{i}$ \\
\hline \multirow{3}{*}{ 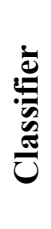 } & 1 & 18 & 9 & 20 & 0 & 57.3 \\
\hline & 2 & 1 & 3 & 0 & 58.3 & 16.7 \\
\hline & 3 & 0 & 0 & 4 & 58.3 & 0 \\
\hline \multicolumn{2}{|c|}{ All } & 19 & 12 & 24 & \multicolumn{2}{|c|}{$e_{0}=54.5 \%$} \\
\hline
\end{tabular}

\subsubsection{Quality assessement using SVM with color features (Lab color model)}

Two types of classifiers are choosen for the present research: SVM linear and SVM kernel with radial basis function. At first a capabilities of linear classifier is investigated. The average error over $\mathrm{k}$-folds is $17.3 \%$ and the confusion matrix of the biggest error is given in Table 3 .

Table 3. The confusion matrix of the biggest error for linear SVM with color features

\begin{tabular}{|c|c|c|c|c|c|c|}
\hline \multirow{2}{*}{\multicolumn{2}{|c|}{$\begin{array}{c}\text { SVM } \\
\text { linear }\end{array}$}} & \multicolumn{3}{|c|}{ True classes } & \multicolumn{2}{|c|}{ errors } \\
\hline & & 1 & 2 & 3 & $\mathbf{g}_{\mathbf{i}}$ & $\mathbf{e}_{i}$ \\
\hline \multirow{3}{*}{ 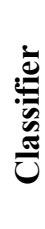 } & 1 & 19 & 2 & 2 & 0 & 17.4 \\
\hline & 2 & 0 & 8 & 5 & 33.3 & 38.5 \\
\hline & 3 & 0 & 2 & 17 & 29.2 & 10.5 \\
\hline \multicolumn{2}{|c|}{ All } & 20 & 12 & 24 & \multicolumn{2}{|c|}{$e_{0}=20 \%$} \\
\hline
\end{tabular}


After that, kernel SVM with radial basis function is modeled. The average error over $\mathrm{k}$-folds is $15.1 \%$ and the confusion matrix of the biggest error is given in Table 4.

Table 4. The confusion matrix of the biggest error for kernel SVM with color features

\begin{tabular}{|c|c|c|c|c|c|c|}
\hline \multirow{2}{*}{\multicolumn{2}{|c|}{$\begin{array}{c}\text { SVM } \\
\text { rbf }\end{array}$}} & \multicolumn{3}{|c|}{ True classes } & \multicolumn{2}{|c|}{ errors } \\
\hline & & 1 & 2 & 3 & $\mathbf{g}_{\mathbf{i}}$ & $\mathbf{e}_{\mathbf{i}}$ \\
\hline \multirow{3}{*}{ 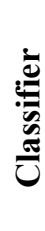 } & 1 & 18 & 1 & 0 & 5.3 & 5.3 \\
\hline & 2 & 1 & 12 & 9 & 7.7 & 45.5 \\
\hline & 3 & 0 & 0 & 15 & 37.5 & 0 \\
\hline \multicolumn{2}{|c|}{ All } & 19 & 13 & 24 & \multicolumn{2}{|c|}{$e_{0}=19.6 \%$} \\
\hline
\end{tabular}

\subsection{Quality assessment using ANN with spectral features}

The spectral characteristics are pre-processed with SNV, MSC and first derivative (Savitzky-Golay) in this sequence. Three principal components PC1, PC2 and PC3 are obtained by principal component analysis (PCA) Fig.8. These principal components are used as an input vector to develop a classifier model. The $\mathrm{k}$-fold cross-validation is applied to the dataset with 5 disjoint subsets from all number of observations.

The BPN neural network structured to model the classifier using principal components is shown in Fig.5. The input layer of the neural network has three inputs corresponding to each of the principal components.

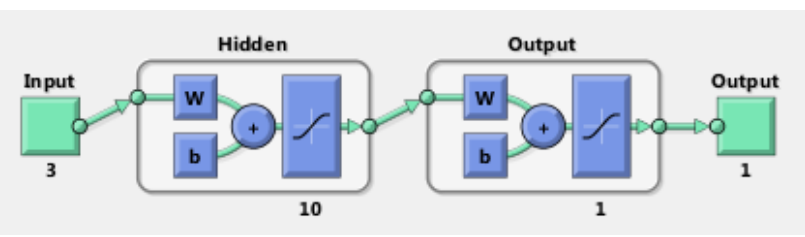

Fig. 5. BPN neural network for spectral feature classification

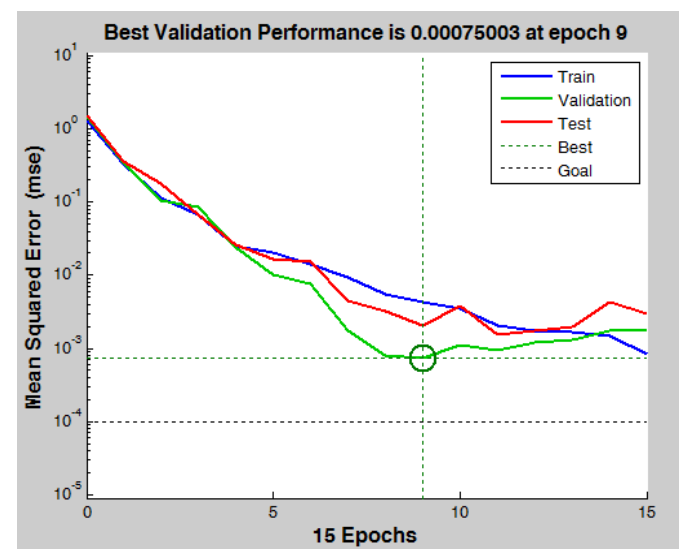

Fig. 6. Training, validation and testing of the best fold for BPN with spectral features
Training, validation and testing of the BPN classifier for the best fold is given on the Fig.6. The average error over $\mathrm{k}$-folds is $4.1 \%$ and the confusion matrix of the biggest error is given in Table 5 .

Table 5. The confusion matrix of the biggest error for BPN with spectral features

\begin{tabular}{|c|c|c|c|c|c|c|}
\hline \multirow{2}{*}{\multicolumn{2}{|c|}{ BPN }} & \multicolumn{3}{|c|}{ True classes } & \multicolumn{2}{|c|}{ errors } \\
\hline & & 1 & 2 & 3 & $\mathbf{g}_{\mathbf{i}}$ & $\mathbf{e}_{\mathbf{i}}$ \\
\hline \multirow{3}{*}{ 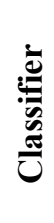 } & 1 & 20 & 0 & 4 & 13.0 & 16.7 \\
\hline & 2 & 0 & 25 & 0 & 0 & 0 \\
\hline & 3 & 3 & 0 & 6 & 40.0 & 33.3 \\
\hline \multicolumn{2}{|c|}{ All } & 23 & 25 & 10 & \multicolumn{2}{|c|}{$e_{0}=12.1 \%$} \\
\hline
\end{tabular}

The PNN neural network structured to model the classifier using principal components is shown in Fig.7. The input layer of the neural network has three inputs corresponding to each of the principal components. The three outputs correspond to the probability of the testing vector to belong to the output class.

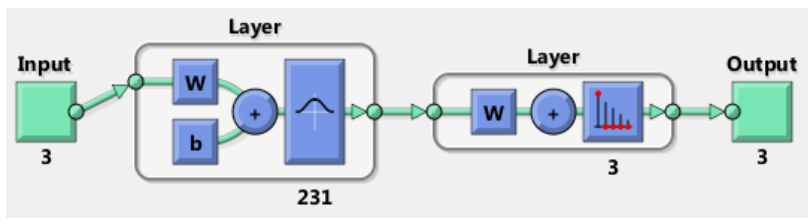

Fig. 7. PNN neural network for spectral feature classification

The results show that after training the PNN classifier, the average error over $\mathrm{k}$-folds is $61.0 \%$ and the confusion matrix of the biggest error is given in Table 6 .

Table 6. The confusion matrix of the biggest error for PNN with spectral features

\begin{tabular}{|c|c|c|c|c|c|c|}
\hline \multirow{2}{*}{\multicolumn{2}{|c|}{ PNN }} & \multicolumn{3}{|c|}{ True classes } & \multicolumn{2}{|c|}{ errors } \\
\hline & & 1 & 2 & 3 & $\mathbf{g}_{\mathbf{i}}$ & $\mathbf{e}_{i}$ \\
\hline \multirow{3}{*}{ 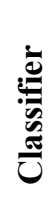 } & 1 & 22 & 26 & 10 & 0 & 62.1 \\
\hline & 2 & 0 & 0 & 0 & 100 & - \\
\hline & 3 & 0 & 0 & 0 & 100 & - \\
\hline \multicolumn{2}{|c|}{ All } & 22 & 26 & 10 & \multicolumn{2}{|c|}{$e_{0}=62.1 \%$} \\
\hline
\end{tabular}




\subsection{Quality assessment using SVM with spectral features}

As mentioned above two types of classifiers, SVM linear and SVM with radial basis function are applied to the dataset, which is compound of transformed spectral characteristics.

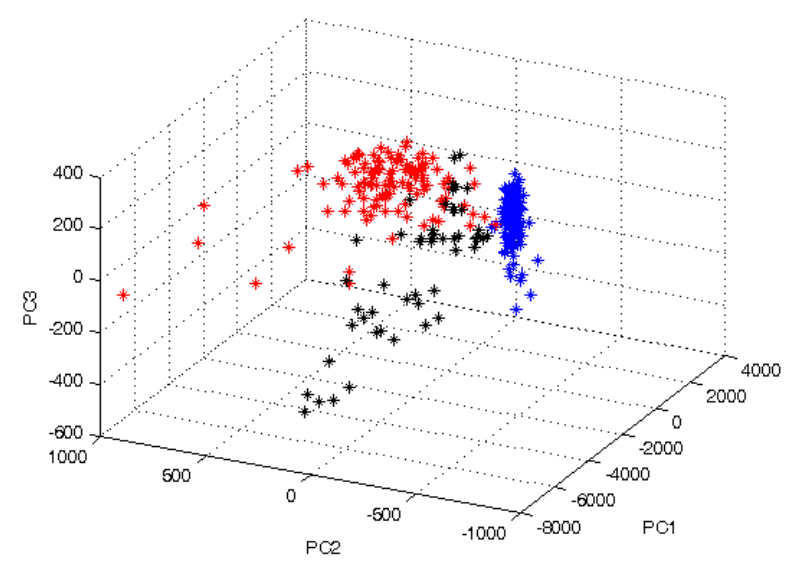

Fig. 8. Three principal components obtained from preprocessed spectral features

The three principal components (PC1, PC2 and PC3) obtained from pre-processed spectral features are shown on Fig.8. The three colors correspond to three classification groups. Training of the classifier is done with a multi-svm algorithm, which uses "one against the rest" method. The training and testing of linear SVM is done over the $5 \mathrm{k}$-fold tests. The average error over kfolds is $3.4 \%$ and the confusion matrix of the biggest error is given in Table 7 . The average error over k-folds when training and testing SVM with radial basis function is $2.4 \%$ and the confusion matrix of the biggest error is given on Table 8 .

Table 7. The confusion matrix of the biggest error for linear SVM with spectral features

\begin{tabular}{|c|c|c|c|c|c|c|}
\hline \multirow{2}{*}{\multicolumn{2}{|c|}{$\begin{array}{c}\text { SVM } \\
\text { linear }\end{array}$}} & \multicolumn{3}{|c|}{ True classes } & \multicolumn{2}{|c|}{ errors } \\
\hline & & 1 & 2 & 3 & $\mathbf{g}_{i}$ & $\mathbf{e}_{\mathbf{i}}$ \\
\hline \multirow{3}{*}{ 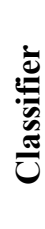 } & 1 & 22 & 1 & 2 & 0 & 12.0 \\
\hline & 2 & 0 & 24 & 0 & 4.0 & 0 \\
\hline & 3 & 0 & 0 & 8 & 22 & 0 \\
\hline \multicolumn{2}{|c|}{ All } & 22 & 25 & 10 & \multicolumn{2}{|c|}{$\mathrm{e}_{\mathrm{o}}=5.3 \%$} \\
\hline
\end{tabular}

Table 8. The confusion matrix of the biggest error for kernel SVM with spectral features

\begin{tabular}{|c|c|c|c|c|c|c|}
\hline \multirow{2}{*}{\multicolumn{2}{|c|}{$\begin{array}{c}\text { SVM } \\
\text { rbf }\end{array}$}} & \multicolumn{3}{|c|}{ True classes } & \multicolumn{2}{|c|}{ errors } \\
\hline & & 1 & 2 & 3 & $\mathbf{g}_{\mathbf{i}}$ & $\mathbf{e}_{\mathrm{i}}$ \\
\hline \multirow{3}{*}{ 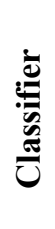 } & 1 & 22 & 0 & 0 & 4.3 & 0 \\
\hline & 2 & 2 & 23 & 0 & 8 & 0 \\
\hline & 3 & 1 & 2 & 10 & 0 & 23.1 \\
\hline \multicolumn{2}{|c|}{ All } & 23 & 25 & 10 & \multicolumn{2}{|c|}{$e_{0}=5.2 \%$} \\
\hline
\end{tabular}

\section{Conclusions}

According to the results for color features, BPN and SVM with radial basis function kernel have the best performance in individual group categorization while PNN has the worst performance. Two types of SVM classifiers have close results with primacy of SVM with radial basis function. The errors here are the following: $\mathrm{e}_{\mathrm{avg}}=17.3 \%$ vs $15.1 \%$ and maximum error $\mathrm{e}_{\max }=20 \%$ vs $19 \%$. The BPN network is well trained with the average error over $\mathrm{k}$-fold $\mathrm{e}_{\mathrm{avg}}=15.1 \%$, and maximum error $\mathrm{e}_{\max }=57.1 \%$, which is a bad sign for the network's ability to generalize. When using PNN network there is no training phase. The results show that this network is unable to classify correctly with errors of $\mathrm{e}_{\text {avg }}=48.2 \%$, and maximum error $\mathrm{e}_{\max }=54.5 \%$. When using color models the SVM with radial basis function have the best accuracy performance. The ability to generalize is obvious, as the average and maximum errors have close values.

The use of spectral characteristics is the second research initiative. The trend here is preserved. The PNN network could not classify correctly again, with following errors: $\mathrm{e}_{\mathrm{avg}}=61.0 \%$ and maximum error $\mathrm{e}_{\max }=$ $62.1 \%$. The interesting thing is that, BPN network performs very well with errors: $\mathrm{e}_{\mathrm{avg}}=4.1 \%$ and maximum error $\mathrm{e}_{\max }=12.1 \%$. The superior from the classifiers is SVM with its two versions. SVM linear classifiers has the following errors: $\mathrm{e}_{\mathrm{avg}}=3.4 \%, \mathrm{e}_{\max }=$ $5.3 \%$ and SVM with rbf kernel: $\mathrm{e}_{\mathrm{avg}}=2.4 \%, \mathrm{e}_{\max }=$ $5.2 \%$. As a conclusion, it could be said that the use of spectral characteristics for apricots quality assessment in a combination with SVM classifier is more appropriate than the use of color features. This is because the classification error is 7 times smaller. All classifiers using spectral features perform well except PNN. Errors are at about 2-5\%. SVM classifiers and BPN neural network in a combination with spectral features are best in evaluating the quality of apricots during the drying process. Classification with color features is an alternative method, which is less complex, cheaper and for some tasks with acceptable errors. 


\section{Acknoledgements}

The study was supported by contract of University of Ruse "Angel Kanchev", "BG05M2OP001-2.009-001101, "Support for the development of human resources for research and innovation at the University of Ruse "Angel Kanchev". The project is funded with support from the Operational Program "Science and Education for Smart Growth 2014 - 2020" financed by the European Social Fund of the European Union.

\section{References}

1. A. Cabrera, J.M. Prieto, Application of artificial neural networks to the prediction of the antioxidant activity of essential oils in two experimental in vitro models, Food Chem., 118, 141 (2010)

2. C. Cimpoiu, V.-M. Cristea, A. Hosu, M. Sandru, L. Seserman, Antioxidant activity prediction and classification of some teas using artificial neural networks, Food Chem., 127, 1323 (2011)

3. V. Farzaneh, A. Ghodsvali, H. Bakhshabadi, M. Ganje, Z. Dolatabadi, I.S.Carvalho, Modelling of the selected physical properties of the Fava bean with various moisture contents using Fuzzy Logic Design, Journal of Food Process Eng., 40, 123 (2016)

4. E. Górska-Horczyczak, M. Horczyczak, D. Guzek, I. Wojtasik-Kalinowska, A. Wierzbicka, Chromatographic fingerprints supported by artificial neural network for differentiation of fresh and frozen pork, Food Control, 73, 237 (2017)

5. M. Hajimahmoodi, M. Khanavi, O. Sadeghpour, M. Ardekani, F. Mazde, M. Khoddami, S. Afzalifard, A. Ranjbar, Application of organic acid based Artificial Neural Network modeling for assessment of commercial vinegar authenticity, Food Anal. Methods, 9, 3451 (2016)

6. A. Hosu, V.M. Cristea, C. Cimpoiu, Analysis of total phenolic, flavonoids, anthocyanins and tannins content in Romanian red wines: Prediction of antioxidant activities and classification of wines using artificial neural networks, Food Chem., 150, 113 (2014)

7. S. Keeratipibul, A. Phewpan, C. Lursinsap, Prediction of coliforms and Escherichia coli on tomato fruits and lettuce leaves after sanitizing by using Artificial Neural Networks, Food Sci. Technol., 44, 130 (2011)

8. L. Pan, Q. Zhang, W. Zhang, Y. Sun, P. Hu, K. $\mathrm{Tu}$, Detection of cold injury in peaches by hyperspectral reflectance imaging and artificial neural network, Food Chem., 192, 2016, pp. 134-141.

9. C. Cevoli, L. Cerretani, A. Gori, M.F. Caboni, T. Gallina Toschi, A. Fabbri, Classification of Pecorino cheeses using electronic nose combined with artificial neural network and comparison with GC-MS analysis of volatile compounds, Food Chem., 129, 1315 (2011)

10. M. León-Roque, L. Abderrahim, S. NuñezAlejos, M. Arribas, L. Condezo-Hoyos, Prediction of fermentation index of cocoa beans (Theobroma cacao L.) based on color measurement and artificial neural networks, Talanta, 161, 31 (2016)

11. J. Rojas, V. Vásquez, Prediction by Artificial Neural Networks (ANN) of the diffusivity, mass, moisture, volume and solids on osmotically dehydrated yacon (Smallantus sonchifolius), Sci. Agropecu., 3, 201 (2012)

12. M. Soto-Barajas, Ma Inmaculada GonzálezMartín, J. SalvadorEsteban, J. HernándezHierro, V. Moreno-Rodilla, A. VivarQuintana, I. Revilla, I. Ortega, R. MorónSancho, B. Curto-Diego, Prediction of the type of milk and degree of ripening in cheeses by means of artificial neural networks with data concerning fatty acids and near infrared spectroscopy, Talanta, 116, 50 (2013)

13. A. Bahmani, S.M. Jafari, S.A. Shahidi, D. Dehnad, Mass transfer kinetics of Eggplant during osmotic dehydration by Neural Networks, Joutnal Food Process. Preserv., 40, 815 (2015)

14. M. Azadbakht, H. Aghili, A. Ziaratban, M.V. Torshizi, Application of artificial neural network method to exergy and energy analyses of fluidized bed dryer for potato cubes, Energy, 120, 947 (2017)

15. P. Khawas, K.K. Dash, A.J. Das, S.C. Deka, Modeling and optimization of the process parameters in vacuum drying of culinary banana (Musa ABB) slices by application of artificial neural network and genetic algorithm, Dry Technol., 34, 491 (2016)

16. T. Amoriello, R. Ciccoritti, M. Paliotta, K. Carbone, Classification and prediction of earlyto-late ripening apricot quality using spectroscopic techniques combined with chemometric tools, Scientia Horticulturae, 240, 310 (2018)

17. M. Khojastehnazhand, V. Mohammadi, S. Minaei, Maturity detection and volume 
estimation of apricot using image processing technique, Scientia Horticulturae, 251, 247 (2019)

18. İ. Sani, Ö. Bülent, Ö. Belgin, Ç. Sarıtepe, H. Aksoy, Rapid, simultaneous and nondestructive assessment of the moisture, water activity, firmness and $\mathrm{SO} 2$ content of the intact sulphured-dried apricots using FT-NIRS and hemometrics, Talanta, 186, 467 (2018)

19. C. Camps, D. Christen, Non-destructive assessment of apricot fruit quality by portable visible-near infrared spectroscopy, LWT - Food Science and Technology, 42, 1125 (2009) 